\title{
SM22 $\alpha$ Promoter Targets Gene Expression to Vascular Smooth Muscle Cells In Vitro and In Vivo
}

\author{
Levent M. Akyürek, ${ }^{1}$ Zhi-Yong Yang, ${ }^{1,2}$ Kazunori Aoki, ${ }^{2}$ Hong San, ${ }^{1}$ \\ Gary J. Nabel, ${ }^{2}$ Michael S. Parmacek ${ }^{3}$ and Elizabeth G. Nabel ${ }^{1}$ \\ ${ }^{1}$ Vascular Biology Branch, National Heart, Lung and Blood Institute, \\ National Institutes of Health, Bethesda, Maryland, U.S.A. \\ ${ }^{2}$ Vaccine Research Center, National Institute of Allergy and Infectious Diseases, \\ National Institutes of Health, Bethesda, Maryland, U.S.A. \\ ${ }^{3}$ Department of Medicine, University of Pennsylvania School of Medicine, \\ Philadelphia, Pennsylvania, U.S.A.
}

Accepted July 28, 2000.

\begin{abstract}
Background: Gene transfer into vascular smooth muscle cells (vsmcs) holds promise for studying the pathogenesis of arterial disorders. However, a potential limitation of vectors with heterologous promoters is organ toxicity resulting from unrestricted transgene expression. Vascular smooth muscle cellspecific gene expression could increase the safety of vectors for vascular diseases.

Materials and Methods: To develop vectors that target gene expression to vsmcs, we constructed vectors encoding human placental alkaline phosphatase (hpAP) and chloramphenicol transferase (CAT) driven by a 441-bp region of the murine $\mathrm{SM} 22 \alpha$ promoter (AdSM22 $\alpha$-hpAP).

Results: Transfection of AdSM $22 \alpha$-hpAP into vascular and nonvascular cells resulted in the expression of alkaline phosphatase (AP) in primary arterial and venous smcs, but not in primary endothelial cells or National Institutes of Health (NIH) $3 \mathrm{~T} 3$ cells. Expression of AP was observed on $32.56 \quad 1.4 \%$ of primary pig vsmcs-infected AdSM22 $\alpha$-hpAP at a multiplicity
\end{abstract}

of infection (MOI) of 500; whereas, infection with AdCMV-hpAP resulted in $1006 \quad 0.0 \%$ expression at a MOI of 250. In vitro, expression from the heterologous cytomegalovirus (CMV) promoter was approximately $10^{3}$-fold higher in vsmcs, compared with the SM22 $\alpha$ promoter. Following introduction of AdSM22 $\alpha$-hpAP vectors into balloon-injured pig arteries, AP recombinant protein was detected in neointimal (2.23 6 $1.14 \%)$ and medial $(0.5660 .21 \%)$ smcs, but not in endothelial or adventitial cells. In contrast, AdCMVhpAP vectors led to AP expression in intimal endothelial and smcs cells (39.14 6 10.09\%) and medial smcs $(2.8461 .05 \%)$. AP expression was not observed in endothelial or vsmcs following transfection with the control vector, adenoviral vector lacking El (AdDE1). Conclusions: The SM22 $\alpha$ promoter programs recombinant gene expression exclusively to vascular smcs in vitro and in vivo. Although expression levels are lower than with heterologous promoters, these vectors may provide a safe and effective tool for gene therapy of vascular diseases.

\section{Introduction}

Proliferation of smooth muscle cells (smcs) and their phenotypic modulation play an integral role in the pathogenesis of vascular prolifera-

Address correspondence and reprint requests to: Elizabeth G. Nabel, National Heart, Lung and Blood Institute/NIH, Building 10, Room 8C103, Bethesda, MD 20892, U.S.A. Phone: 301-496-1518; Fax: 301-402-7560;

E-mail: enabel@nih.gov tive disorders, such as transplant arteriosclerosis, restenosis, and failure of bypass grafts (1). Genetic manipulation of vascular smooth muscle cells (vsmcs) has been used to study growth regulation, angiogenesis, thrombosis, and lipoprotein metabolism in the vasculature (2). Adenoviral vectors efficiently transduce both resting and replicating smcs in vivo. However, these vectors currently utilize viral promoters that program unrestricted ectopic gene expression 
in many cells and tissues, including the liver and lung. Tissue-specific, promoter-driven vectors may offer an enhanced safety profile by reducing ectopic expression in vital organs.

Vascular smcs exhibit phenotypic plasticity and undergo transition between proliferative and differentiated states; whereas, skeletal and cardiac muscle cells differentiate irreversibly (3). The differentiated state of vascular smcs is marked by a number of smc-specific proteins, including caldesmon, calponin, desmin, smooth muscle $\alpha$-actin, smooth muscle-myosin heavy chain (SM-MHC), SM22 $\alpha$ and telokin (4). Compared with other smc-specific products, SM22 $\alpha$ offers advantages as a phenotypic marker. SM22 $\alpha$ is an early marker and is expressed exclusively in visceral and vascular smcs. However, its expression in embryonic tissue is more widespread and detected in heart, skeletal muscle cells, and myotomal compartments (5). Using a series of promoter deletions, a 441-bp (6) and 445-bp $(7,8)$ region of 59 -flanking sequence in the murine SM22 $\alpha$ promoter has been shown to be necessary and sufficient to program high-level transgene expression in smc lineages.

Transgene expression with heterologous promoters results in ubiquitous expression; whereas, tissue-specific transcriptional gene regulation generally limits recombinant expression to specific cells and tissues. For application to human clinical protocols, it may be preferable to selectively express transgenes within target cells in order to avoid possible systemic toxicities. We tested the hypothesis that the SM22 $\alpha$ promoter would restrict transgene expression exclusively to vsmcs in vitro and in vivo. To examine this hypothesis, transgene expression driven by a smc-specific promoter was investigated in cell culture and a large animal model of vascular injury. We chose to investigate SM22 $\alpha$, since SM22 $\alpha$ is not downregulated after embryonic development or in a cell-cycle dependent fashion suggesting a role for the protein in both contractile and synthetic state, in contrast to the other smc-specific proteins. We de-monstrated that reporter gene expression was driven exclusively in arterial and venous smcs in vitro and in vivo, although at lower levels, compared with the heterologous cytomegalovirus (CMV) promoter. In contrast to the CMV promoter, SM22 $\alpha$-driven recombinant AP expression was not observed in organs outside the vasculature, including the liver or the lung of pigs following gene transfer, sug- gesting a favor-able safety profile. Thus, transgene expression can be restricted exclusively to smcs by a SM22 $\alpha$ promoter and this promoter provides a promising strategy to deliver selectively therapeutic genes to vsmcs in vivo.

\section{Materials and Methods}

\section{Plasmids}

The pAdMCS-loxP plasmid, an eukaryotic expression vector containing the left-hand sequence (59L-ITR) and packaging signal from 0 to 1 map units of the Ad5genome, but not ElA and ElB, and a 34-bp oligonucleotide loxP sequence, was used as a backbone (9). A 441-bp fragment of the SM22 $\alpha$ promoter with a Sal l site at the 59end and Hind III and Xho I site at the 39 end was synthesized from murine genomic DNA by polymerase chain reaction (PCR) (9). The plasmid pAdSM22 $\alpha$-loxP contains the 441-bp part of the SM22 $\alpha$ promoter (Xho I site), the 231-bp bovine growth hormone polyadenylation signal ( $\mathrm{Xba}$ I site), and the 471-bp human 4F2 enhancer (Not I site)(10). Human placental alkaline phosphatase (hpAP) and chloramphenicol acetyltransferase (CAT) genes from the pBS-hpAP (2) and pVR1332-CAT (11) were cloned into pAdSM22 $\alpha$-loxP (EcoRV and Xho I sites) to construct the pAdSM22 $\alpha$-hpAP and pAdSM $22 \alpha$-CAT, respectively.

\section{Recombinant Adenoviral Vectors}

Replication-deficient recombinant adenoviral vectors encoding alkaline phosphatase (AP) and CAT were prepared by Cre/loxP-mediated recombination (12) of pAdSM22 $\alpha$-hpAP and pAd-SM22 $\alpha$-CAT with a sub360 adenoviral cosmid, an Ad5 derivative with a deletion in the $\mathrm{E} 3$ region. There was a deletion in the E1A and ElB region, impairing the ability of these viruses to replicate and transform nonpermissive cells. 59 L-ITR was deleted in the adenoviral cosmid, making it incapable of packaging. Recombinant adenoviral vectors under control either of the CMV or SM22 $\alpha$ promoter were prepared as described (13). Purified DNA was transfected into 293 cells using Lipofectamine following manufacturer suggested protocol. After 7 to 9 days, the cytopathic effect was observed and the cells were harvested. To generate higher titer viral stocks, 293 cells were infected and harvested with the resultant viruses purified by cesium chloride. Purified viral stocks 
were diluted for storage in a $13 \%$ glycerol/ phosphate-buffered saline (PBS) solution to yield a final concentration of $1310^{12}$ viral particles $/ \mathrm{ml}$. The adenoviral vector lacking El (AdDEl) and containing no insert was used as a control vector.

\section{Southern Blots Analysis}

Genomic DNA was extracted from 293 cells infected with the crude cell lysate incubated for 3 days and linearized with Pme I. One microgram of digested DNA was fractionated on a $0.4 \%$ agarose gel, transferred onto a nylon membrane, and hybridized with ${ }^{32} \mathrm{P}$-labeled $\mathrm{El}$ probe in $5 \mathrm{X}$ Denhardt's solution [0.1\% sodium dodecyl sulfate (SDS), $5 \mathrm{X}$ sodium citrate sodium chloride sodium phosphate EDTA (SSPE)] and 100 $\mu \mathrm{g} / \mathrm{ml}$ of salmon testis DNA at $658 \mathrm{C}$ for $16 \mathrm{hr}$. The filters were then washed in $0.1 \mathrm{X}$ standard saline citrate (SSC) and $0.1 \%$ SDS at 658 .

\section{AP Staining and CAT Assay}

Porcine aortic smcs (PASMC), porcine jugular vein smcs (PJVSMC), mouse aortic smcs (MASMC), and porcine aortic endothelial cells (PEC) were grown in M199 supplemented with penicillin and streptomycin plus $10 \%$ fetal bovine serum (FBS). National Institutes of Health 3T3 cells were grown in Dulbecco's modified Eagle's medium (DMEM) supplemented with penicillin and streptomycin plus $10 \%$ calf serum. Cells were infected with adenoviral vectors encoding either AP or CAT in $2 \%$ serum for $1 \mathrm{hr}$ at multiplicity of infection (MOIs) from 0 to 1000, where 1 MOI equaled 100 viral particles per cell or 100 viral particles per plaque-forming unit. Two days after infection, cells were either fixed and stained for AP or harvested for CAT assay as described $(2,11)$.

\section{Gene Transfer In Vivo}

To assess the transduction efficacy of AP gene driven either by the SM22 $\alpha$ or CMV promoter, we used a standard large animal model of vascular disease, injured pig arteries. Femoral arteries were injured and infected with adenoviral vectors with a double-balloon catheter as described (13); whereas, control arteries were injured and infected with AdDEl (n 56 arteries in each group). Four days after injury and infection, each artery was processed in an identical manner by division into eight segments and paraffin-embedded. For organ toxicity studies, all major organs and vessels were harvested. All samples were sectioned and stained with hematoxylin and eosin (HEE) and AP. The number of AP-positive cells and the total number of cells were analyzed in the intima and media regions by two independent reviewers in a blinded fashion. To determine which cells in the intima expressed the recombinant AP gene, serial cross sections were stained as described with monoclonal antibodies against either smooth muscle-specific $\alpha$-actin or von Willebrand's factor VIII to identify smcs or endothelial cells, respectively (13). All animal experiments were performed with the approval of the University of Michigan Committee in the Use and Care of Animals.

\section{Statistical Analysis}

Statistical calculations were performed using the StatView ${ }^{\mathrm{TM}}$ (Abacus Concepts Inc., Berkeley, CA). Data are presented as the mean 6 standard error of the mean (SEM). Analyses of variance (ANOVA) with contingency tables were used for comparisons among multiple groups. If this test showed a significant heterogeneity of all groups, we further compared each group by applying the Bonferroni-Dunn test. A $p$-value of , 0.05 was considered statistically significant.

\section{Results}

Ectopic Expression Driven by 441-bp of the SM22 $\alpha$ Promoter was Restricted to smes In Vitro

Adenoviral vectors driven by the SM22 $\alpha$ (441) promoter encoding reporter genes, AP and CAT, were constructed by Cre/loxP recombination (Fig. 1A). No wild-type adenoviral DNA was detected using an El region as a probe for Southern blot analysis (Fig. 1B). Furthermore, no replication complement, virus could be detected by TaqMan PCR method (Perkin Elmer, Norwalk, CT) using El-primer (14). Infection of A549 cells with 1000 MOI of the adenoviral vectors did not produce a cytopathic effect or subsequent viral plaque formation.

Recombinant AP expression of the AP reporter gene encoded by AdSM22 $\alpha$-hpAP was observed in both primary arterial PAMSC and MASMC and venous PJVSMC smcs, but not in fibroblast (NIH 3T3) or porcine aortic endothelial cell (PEC). In contrast, AP expression was detected following infection with AdCMVhpAP in both smc and non-smc replicate cultures 


\section{A}
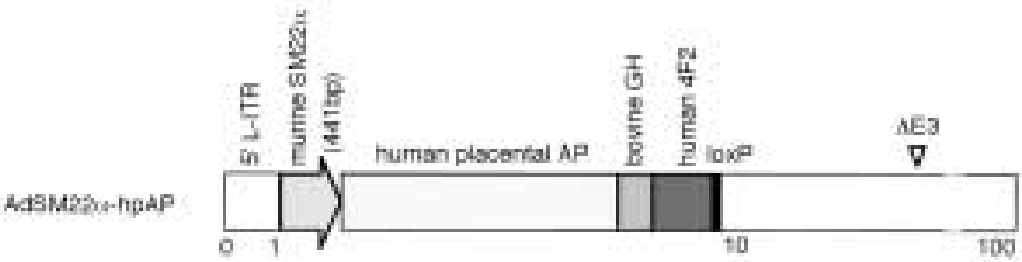

ACMN-haAP
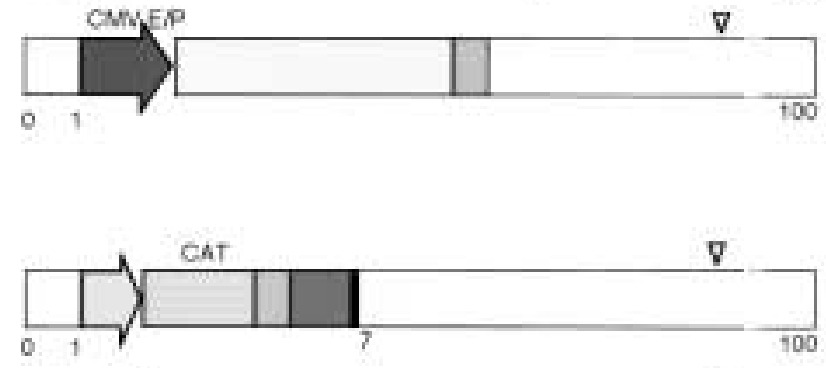

CAT

AGCMV-CAT

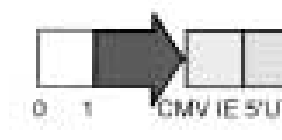

Fig. 1. Smooth muscle cell (SMC)-specific adenoviral vectors. (A) Structure of the adenoviral vectors, AdSM22 $\alpha$-hpAP and AdSM22 $\alpha$-CAT, encoding either human placental alkaline phosphatase (hpAP) or chloramphenicol transferase (CAT), respectively. Recombinant cytomegalovirus (CMV) adenoviral vectors drive reporter gene expression under control of the cytomegalovirus immediate early gene promoter/enhancer (CMV E/P) and/or intron (CMV IE 59UT). The adenoviral cosmid Sub360 genome has a single loxP sequence and deletion in the El and E3 regions (DE3), generating

(Fig. 2). Infection with AdCMV-hpAP at 250 MOI resulted in nearly $100 \%$ of AP expression in all cell lineages investigated. Recombinant hpAP expression was lower following transfection with AdSM22 $\alpha$ vectors: $32.561 .44 \%$ of the PASMCs expressed AP following infection with a MOI of 500 (Fig. 3A). Increasing the MOI to 1000 did not increase the percentage of transfected vsmc significantly. The level of CAT activity following infection with AdCMV-CAT was several thousand-fold $(7,2426$ 2,884 ) greater, compared with that of infection with AdSM22 $\alpha$-CAT in cultured porcine smcs $(p, 0.0001$; Fig. 3B).

Vascular Gene Delivery with AdSM22 $\alpha$-hpAP to Injured Arteries Confirms smc Specificity

Following gene transfer to injured arteries, ectopic AP expression was not observed in control
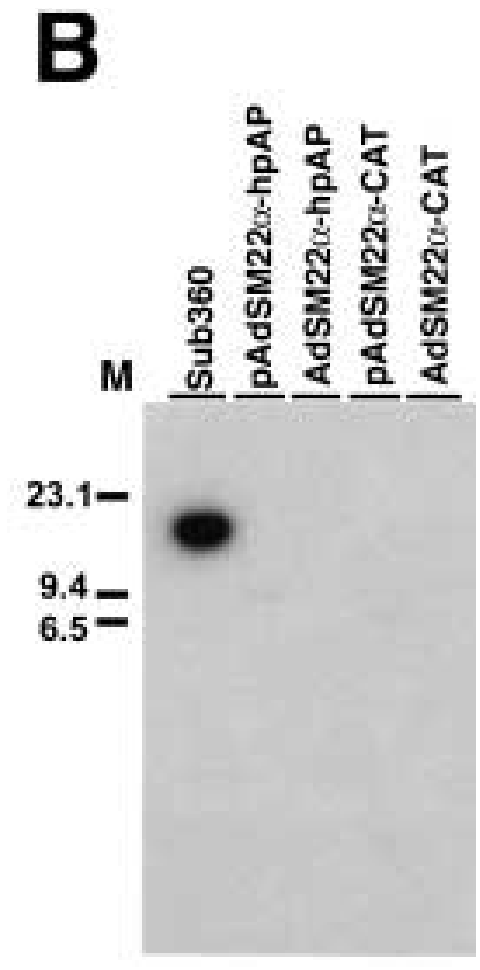

Map units

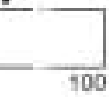

$\nabla$
B

replication-defective adenovirus. The shuttle plasmid and adenoviral cosmid were recombined by Cre-loxP. (B) Southern blots analyses of AdSM $22 \alpha$-hpAP and AdSM22 $\alpha$-CAT. High molecular weight DNA was prepared from cells, and hybridized to the ${ }^{32} \mathrm{P}$-radiolabelled El cDNA probe under high stringency conditions. Respective shuttle plasmids and Ad5Sub360 adenoviral genomic cDNA served as negative and positive controls, respectively. Size markers (M) in kilobases are shown to the left of the blot.

(AdDE1) infected arteries (Fig. 4A). Gene delivery of AdSM22 $\alpha$-hpAP vectors resulted in recombinant AP expression exclusively in intimal and medial vsmcs (Figs. $4 B$ and $4 C$ ). In these arteries, AP expression in sequential sections colocalized with $\alpha$-actin-positive smcs and not von Willebrand's factor-positive endothelial cells. In contrast, when AdCMVhpAP vectors were introduced into injured arteries, AP expression was evident in the intima 4 days later (Figs. 4D and 4E). Medial AP-expressing cells were in close proximity to the internal elastic lamina. Rare AP-positive cells could also be seen in the adventita after infection with AdCMV-hpAP. The number of cells expressing AP in the intima was greater in AdCMV-hpAP infected segments, compared with that of arterial segments infected with AdSM22 $\alpha$-hpAP ( $p \quad 5 \quad 0.007 ; 396 \quad 10.1 \%$ vs. $2.261 .1 \%$, respectively; Fig. 5). In the medial 


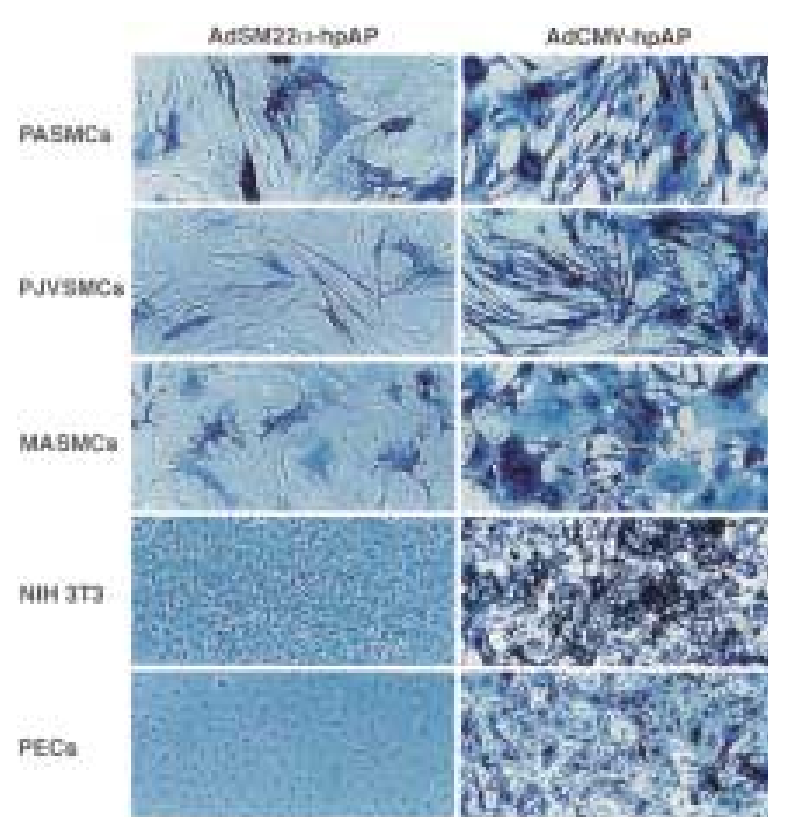

Fig. 2. Smooth muscle cell (SMC) specifity of AdSM22 $\alpha$-hpAP. Primary porcine aortic smcs (PASMC), jugular vein smcs (PJVSMC), mouse aortic smcs (MASMC), porcine aortic endothelial cells (PEC), and murine fibroblasts (NIH93T3) were infected with a viral titer of 250 or 500 (MOI) of AdSM $22 \alpha$-hpAP or AdCMV-hpAP. Two days after infection, cells were fixed and stained for alkaline phosphatase (AP) activity. Ectopic AP expression encoded by AdSM $22 \alpha$-hpAP was restricted to primary smcs and was not seen in endothelial cells or fibroblasts; whereas, the AdCMV-hpAP adenoviral vector programed AP expression in both smooth and non-smooth muscle cell lineages.

layer, the number of AP-expressing cells was increased in the AdCMV-hpAP infected arterial segments. However, this difference was not statistically significant, compared with the AdSM22 $\alpha$-hpAP infected arterial segments ( $p 5$ $0.07 ; 2.86 \quad 1.0 \%$ vs. $0.66 \quad 0.2 \%$, respectively; Fig. 5). To investigate systemic effects after intra-arterial gene transfer with AdSM22 $\alpha$ hpAP, major organs were examined for inflammation and transgene expression. Inflammatory infiltrates were not observed in these organs. The systemic spread of vectors following intra-arterial gene transfer was minimal, as alkaline phosphatase expression was not observed in the organs.

\section{Discussion}

We generated vectors encoding reporter genes under the transcriptional control of the smcspecific SM22 $\alpha$ promoter and demonstrated that the product of a recombinant gene encoded by these vectors can be regulated in smc-specific fashion in vitro and in an in vivo model of vascular injury. Transgene expression driven by the SM $22 \alpha$ promoter was restricted to arterial and venous smc lineages, and no expression was observed in endothelial cells or fibroblasts. However, the level of expression in smcs was significantly lower than expression driven by the CMV promoter. In addition, the lack of transgene expression in major organs following systemic administration of the SM22 $\alpha$ vectors offered better safety by restricting ectopic expression in non-smc containing tissues.

In response to physiological and pathophysiological stimuli, smcs can modulate their phenotype by down-regulating a set of contractile protein genes. These smc-specific marker genes are regulated at the levels of transcription and splicing (4). In contrast to other gene products, SM22 $\alpha$ appears to be constitutively expressed at high levels in both quiescent and proliferating smcs (6). The SM22 $\alpha$ gene product is a $22-\mathrm{kDa}$ protein with structural homology to the vertebrae thin myofibrillar regulatory protein, calponin, and Drosphila mp20 (15). The human SM22 $\alpha$ gene cytogenetically localizes to chromosome $11 \mathrm{q} 23.2$ (16). It is a $6.2-\mathrm{kb}$ single copy gene composed of five exons. SM22 $\alpha$ expression is restricted to smooth muscle cells in adult vertebrae; whereas, its expression in embryonic tissue is detected in heart, skeletal muscle cells, and myotomal compartments (5). In vitro analysis of differentiated versus proliferating rat smcs by mRNA differential display reveals that SM22 $\alpha$ is expressed at high levels in freshly dispersed cells, but it is down-regulated, dramatically in late passage cells (17).

The promoter of SM22 $\alpha$ has been cloned from rat (18), mouse (6), and human (16) DNA. Despite the fact that the gene encoding SM22 $\alpha$ is expressed in both vascular and visceral SMCs in vivo, the 441-bp SM22 $\alpha$ promoter restricts gene expression to arterial SMCs in transgenic mice. The 441-bp promoter contains two CArG boxes, or SREs that bind specifically to the MADS box transcription factor SRF $(19,20)$. Interestingly, SRF has been shown to play an important role in regulating cardiac and skeletal muscle-specific gene expression. In addition, binding sites for Spl, YYl and CREB/ATFl family members have been identified in the SM22 $\alpha$ promoter (9).

A $16-\mathrm{kb}$ fragment of the SM-MHC promoter-intron directs lacZ expression in all smc 


\section{A}

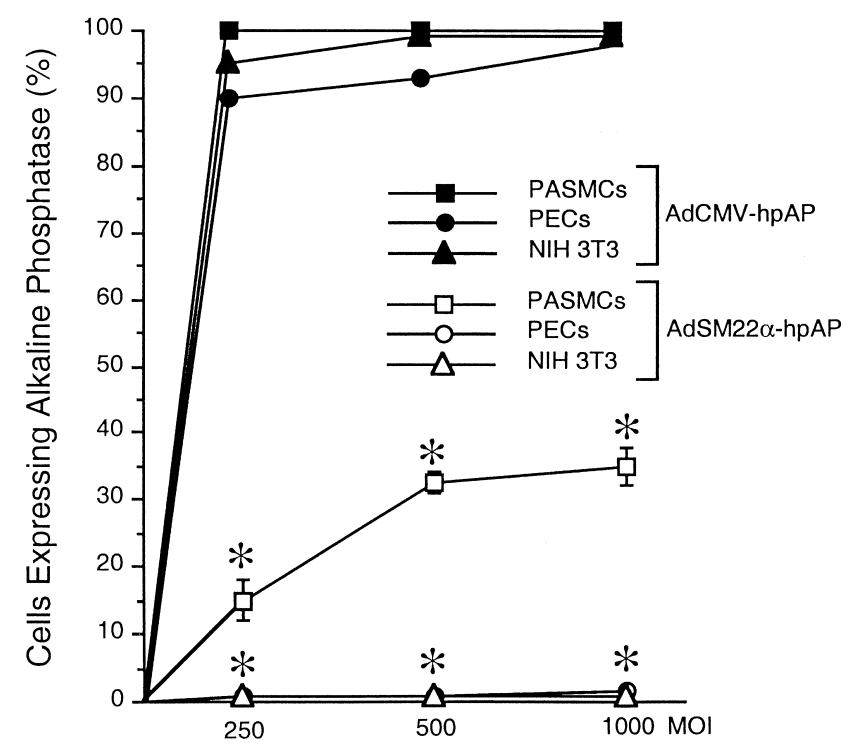

Fig. 3. Promoter activity. (A) Comparison of the activity of adenovirus AdSM22 $\alpha$-hpAP and AdCMV-hpAP control virus in primary cell cultures of porcine aortic smooth muscle cells (PASMC), endothelial cells (PEC), and murine fibroblasts (NIH9 3T3). The cells were infected with 250, 500 and 1000 MOI of either AdSM22 $\alpha$-hpAP or AdCMVhpAP. Percentage of cells expressing AP activity was quantitated $48 \mathrm{hr}$ after infection. Data are expressed as the mean percentage of cells expressing AP

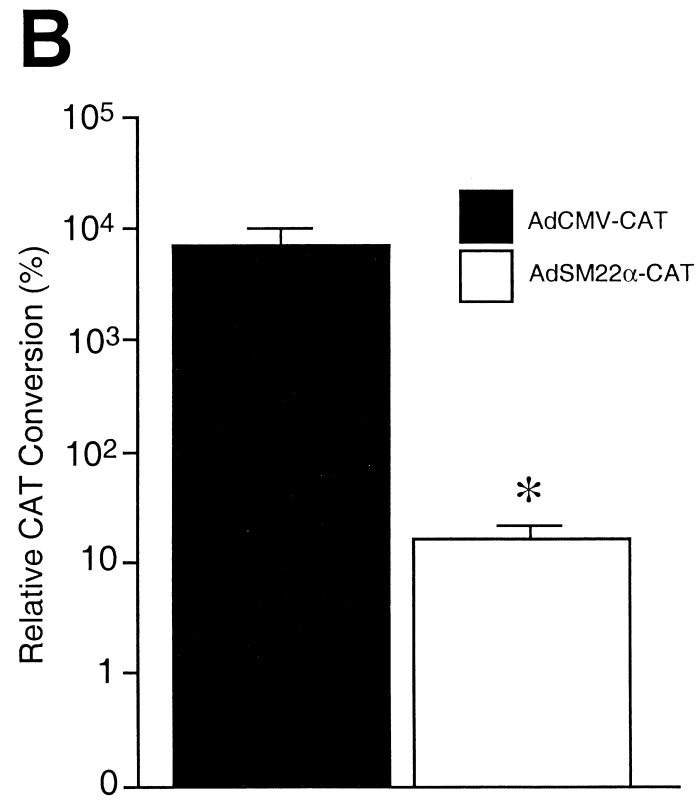

activity 6 standard error of the mean (SEM). (B) Comparison of chloramphenicol transferase (CAT) activity in the PASMCs infected with either AdSM22 $\alpha$-CAT or AdCMV-CAT control vector in vitro. CAT conversion activity was quantitated $48 \mathrm{hr}$ after infection. Level of activity between CMV and SM22 $\alpha$ promoters was compared in each group by analysis of variance. ${ }^{*} p, 0.0001 . \mathrm{hpAP}$, human placental alkaline phosphatase; CMV, cytomegalovirus.

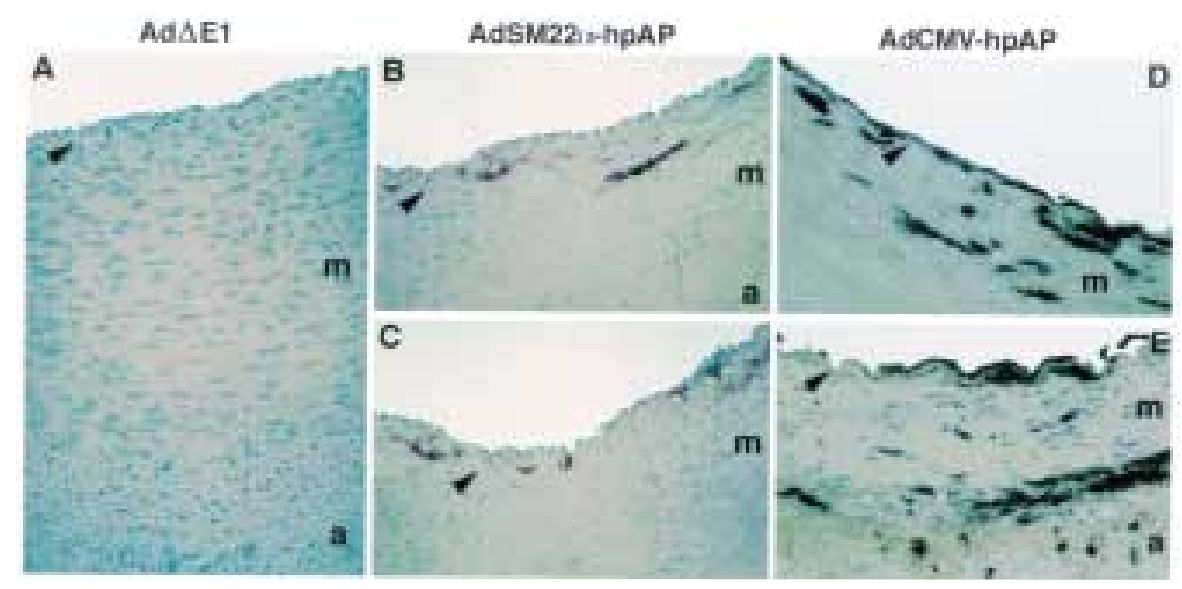

Fig. 4. Alkaline phosphatase (AP) expression in injured and infected porcine femoral arteries. (A) Following infection with adenovirus AdSM22 $\alpha$-hpAP, only intimal and medial smooth muscle cells ectopically expressed AP (B and C). In contrast, in addition to vascular smooth muscle cells (smcs), luminal endothelial and adventitial cells expressed recombinant AP protein after infection AdCMV-hpAP (D and E). The sections were slightly counterstained by methyl green. Arrowhead indicates internal elastic lamina. Original magnification is $200 \mathrm{X}$. hpAP, human placental alkaline phosphatase; CMV, cytomegalovirus. m 5 media, a 5 adventitia 


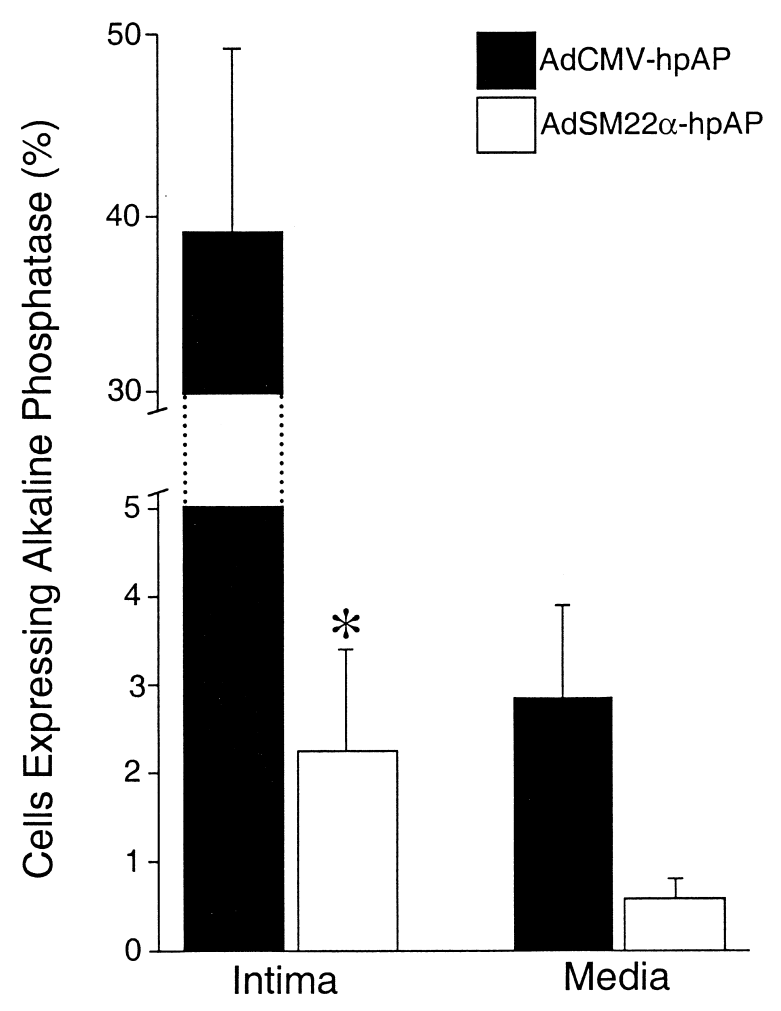

Fig. 5. Expression of alkaline phosphatase (AP) in vivo. Comparison of the number of intimal and medial AP positive cells between the femoral arteries infected either with adenoviruses AdSM $22 \alpha$-hpAP or AdCMV-hpAP four days after injury and infection in the pig. In each arterial segment, the number of AP-positive cells in the intima was counted in four randomly chosen areas; whereas, AP-positive medial cells were counted in the entire section. The total number of cells was obtained from the identical areas of hematoxylin and eosin (H\&E) stained sections. Data is expressed as mean 6 standard error of the mean (SEM) values. The number of transduced cells was compared by analysis of variance. ${ }^{*} p, 0.01$. hpAP, human placental alkaline phosphatase; CMV, cytomegalovirus.

types, including arterial, venous, airway, and visceral smcs in adult transgenic mice (21). This region confers complete smc specificity in vivo. However, SM22 $\alpha$ promoter constructs have not been reported to direct reporter expression in the veins of transgenic mice (8). Interestingly, the SM22 $\alpha$ promoter activity has not been tested in venous smcs in vitro. In this study, AP recombinant protein expression encoded by AdSM22 $\alpha$-hpAP was evident in vein smcs. This difference may be explained by episomal adenoviral DNA. In contrast, the DNA is integrated into the host genome in transgenic mice, where its transcriptional activity may be modulated by alterations in chromatin structure. Although we confirmed the smc- specificity of the SM22 $\alpha$ (441-bp) promoter, a low level of AP expression was noted in smc lineages, in contrast to the high expression of $\beta$-gal reporter gene encoded within AdSM22 $\alpha$ lacZ, as reported by Kim et al (22). The level of $\beta$-gal expression encoded within AdSM22 $\alpha$ lacZ has been reported to be comparable to that of AdCMV-lacZ. We, therefore, applied a quantitative analysis to find out the difference in CAT expression encoded either by the CMV or SM $22 \alpha$ promoter. The CMV promoter was several thousand-fold stronger in cultured smcs.

The phenotypic modulation of smcs has been implicated in the pathogenesis of human vascular disorders (1). A great deal of evidence suggests that the paradigm of a "contractile versus synthetic SMC phenotypes" that was based upon the behavior of primary vascular SMCs in vitro, does not reflect the full spectrum of SMC phenotypes observed in the vessel wall. SMCs are frequently observed during embryonic development and in vascular proliferative syndromes that express high levels of contractile protein isoforms as well as extracellular matrix. It is noteworthy the SM22 $\alpha$ is expressed at relatively high levels within the fibrous cabs of atherosclerotic lesions, but SM22 $\alpha$ is not detectable in underlying neointimal $\mathrm{SMCs}^{23}$. Given the critical role that the fibrous cap plays in mediating plaque stability, it would be of interest to determine whether the RdAV-encoded transgene under the transcriptional control of the SM22 $\alpha$ promoter would transduce SMCs within the fibrous cap of complex atherosclerotic lesions observed in ApoE-deficient and/or ApoBec $^{-/-}$, LDL receptor ${ }^{-/-}$mice. We would predict that an RdAV-encoded transgene under the control of the SM22 $\alpha$ promoter would successfully transduce these cells.

Phenotypic modulation of smcs plays an important role not only in vascular disorders, but also in leiomyogenic tumorigenicity. We showed earlier that systemic administration of an adenoviral vector driven by a SM22 $\alpha$ (441bp) promoter and encoding a non-cleavable Fas ligand can suppress the growth of tumors of smooth muscle origin in vivo without causing systemic organ toxicity (24). This indicates that SM22 $\alpha$-promoter driven adenoviral gene delivery can achieve therapeutic levels in vivo and enhances the safety profile by restricting the recombinant expression to smcs. Tissue-specific promoters drive low level transgene expression, compared with heterologous viral promoters. However, the low level expression of 
SM22 $\alpha$ promoter may be circumvented by a therapeutic approach that enhances the efficacy of gene transfer by inducing bystander effect in the arterial wall. It has been reported that as low as $10 \%$ of thymidine kinase (TK)-transduced cells can cause the death of an entire smc population in vitro (13). Although bystander effect may overcome the smc-restricted specificity in neighboring vascular cells of different types to some degree, it may be minor. Nevertheless, TK transduction by a SM22 $\alpha$ adenoviral vector and subsequent prodrug administration would allow the endothelium to resurface, thereby reducing thrombogenecity and vasoconstriction. This may represent an alternative strategy to specifically target replicating smc in the vascular wall.

In this study, the SM22 $\alpha$ (441-bp) promoter drives transgene expression exclusively in vascular smcs in vitro. In an animal model of vascular injury, reporter gene expression is restricted to smcs following adenoviral gene transfer. Provided that expression levels are high enough to achieve therapeutic efficacy, SM22a-promoter driven adenoviral vectors may offer a tool to specifically target smcs for the treatment of proliferative disease in the vessel wall. Of equal importance, by limiting transgene expression in smc lineages and tissues, adenoviral gene delivery using a SM22 $\alpha$ promoter may provide added safety for human studies in vascular diseases.

\section{Acknowledgments}

We gratefully acknowledge $\mathrm{Xu}$ Lingling for technical expertise for histology and Lisa Nadzam for manuscript preparation. This study was supported in part by NIH R01 56915 to M.S.P. M.S.P. is an Established Investigator of the American Heart Association.

\section{References}

1. Ross R. (1993) The pathogenesis of atherosclerosis: a perspective for the 1990s. Nature 362: 801-809.

2. Nabel EG, Plautz G, Nabel GJ. (1990) Sitespecific gene expression in vivo by direct gene transfer into the arterial wall. Science 249: 1285-1288.

3. Schwartz SM, deBlois D, O'Brien ER. (1995) The intima. Soil for atherosclerosis and restenosis. Circ. Res. 77: 445-465.
4. Sobue K, Hayashi K, Nishida W. (1999) Expressional regulation of smooth muscle cell-specific genes in association with phenotypic modulation. Mol. Cell Biochem. 190:105-118.

5. Li L, Miano JM, Cserjesi P, Olson EN. (1996) SM22 $\alpha$, a marker of adult smooth muscle, is expressed in multiple myogenic lineages during embryogenesis. Circ. Res. 78: 188-195.

6. Solway J, Seltzer J, Samaha FF, et al. (1995) Structure and expression of a smooth muscle cell-specific gene, SM22 $\alpha$. J. Biol. Chem. 270: 13460-13469.

7. Li L, Miano JM, Mercer B, Olson EN. (1996) Expression of the SM22 $\alpha$ promoter in transgenic mice provides evidence for distinct transcriptional regulatory programs in vascular and visceral smooth muscle cells. J. Cell Biol. 132: 849-859.

8. Moessler H, Mericskay M, Li Z, Nagl S, Paulin D, Small JV. (1996) The SM22 promoter directs tissue-specific expression in arterial but not in venous or visceral smooth muscle cells in transgenic mice. Development 122: 2415-2425.

9. Kim S, Ip HS, Lu MM, Clendenin C, Parmacek MS. (1997) A serum response factor-dependent transcriptional regulatory program identifies distinct smooth muscle cell sublineages. Mol. Cell Biol. 17: 2266-2278.

10. Karpinski BA, Yang LH, Cacheris P, Morle GD, Leiden JM. (1989) The first intron of the 4F2 heavy-chain gene contains a transcriptional enhancer element that binds multiple nuclear proteins. Mol. Cell Biol. 9: 2588-2597.

11. Sihmari RD, Yang ZY, Ling X, et al. (1998) Requirements for enhanced transgene expression by untranslated sequences from the human cytomegalovirus immediate-early gene. Mol. Med. 4: 700-706.

12. Aoki K, Barker C, Danthinne $X$, Imperiale MJ, Nabel GJ. (1999) Efficient generation of recombinant adenoviral vectors by Cre-lox recombination in vitro. Mol. Med. 5: 224-231.

13. Ohno T, Gordon D, San H, et al. (1994) Gene therapy for vascular smooth muscle cell proliferation after arterial injury. Science 265: 781-784.

14. Zhang WW, Koch PE, Roth JA. (1995) Detection of wild-type contamination in a recombinant adenoviral preparation by PCR. Biotechniques 18: 444-447.

15. Ayme-Southgate A, Southgate R, Saide J, Benian GM, Pardue ML. (1995) Both synchronous and asynchronous muscle isoforms of projectin (the Drosophila bent locus product) contain functional kinase domains. J. Cell Biol. 128: 393-403.

16. Camoretti-Mercado B, Forsythe SM, LeBeau MM, et al. (1998) Expression and cytogenetic localization of the human SM22 gene (TAGLN). Genomics 49: 452-457.

17. Shanahan CM, Weissberg PL, Metcalfe JC. 
(1993) Isolation of gene markers of differentiated and proliferating vascular smooth muscle cells. Circ. Res. 73: 193-204.

18. Kemp PR, Osbourn JK, Grainger DJ, Metcalfe JC. (1995) Cloning and analysis of the promoter region of the rat SM22 $\alpha$ gene. Biochem. J. 310: 1037-1043.

19. Zilberman A, Dave V, Miano J, Olson EN, Periasamy M. (1998) Evolutionarily conserved promoter region containing $\mathrm{CArG}^{*}$-like elements is crucial for smooth muscle myosin heavy chain gene expression. Circ. Res. 82: 566-575.

20. Mack CP, Thompson MM, Lawrenz-Smith S, Owens GK. (2000) Smooth muscle $\alpha$-actin CArG elements coordinate formation of a smooth muscle cell-selective, serum response factor-containing activation complex. Circ. Res. 86: 221-232.

21. Madsen CS, Regan CP, Hungerford JE, White SL,
Manabe I, Owens GK. (1998) Smooth musclespecific expression of the smooth muscle myosin heavy chain gene in transgenic mice requires $5^{\prime}$ flanking and first intronic DNA sequence. Circ. Res. 82: 908-917.

22. Kim S, Lin H, Barr E, Chu L, Leiden JM, Parmacek MS. (1997) Transcriptional targeting of replication-defective adenovirus transgene expression to smooth muscle cells in vivo. J. Clin. Invest. 100: 1006-1014.

23. Shananhan CM, Cary NRB, Metcalfe JC, Weissberg PL. (1994) High expression of genes for calcification-regulating proteins in human atherosclerotic plaques. J Clin Invest 93: 23932402.

24. Aoki K, Akyürek LM, San H, et al. (2000) Restricted expression of an adenoviral vector encoding Fas ligand (CD95L) enhances safety for cancer gene therapy. Mol. Therapy 1: 555-565. 